\title{
QUERIES-REPLIES
}

6. Scarce Mathematical Tables (Q 2; QR 5).-Mr. R. D. Brown Jr. of Blue Bell, Montgomery Co., Penna., has a copy of $A$, which he kindly loaned to us, so that a film might be made for the library of Brown University, and checks with other tables might be effected. The title-page (corrected) is as follows: Calcul des Rouages $\mid$ par $\mid$ Approximation.|Nouvelle Méthode|par|Achille Brocot|Horloger.|Paris|Achille Brocot, 6, rue du Parc-Royal |Londres $\mid$ Lemaitre et Bergmann, 65, Cannon Street (West)| near St. Paul's Cathedral|1862. 97 p. $16.7 \times 26.1 \mathrm{~cm}$. On p. 47 is the half title: "Table de conversion en Décimales des Fractions ordinaires. A l'usage du Calcul des Rouages par Approximation. Méthode Nouvelle." This is exactly the title of $B$ which contained 51 pages, precisely the number of the pages 47-97. This suggests that these pages of $A$ were reprinted to form $B$.

In $M T A C$, p. 22, it was noted that the Hütte table gives the decimal equivalent, to $11 \mathrm{D}$, of all proper fractions whose positive numerators and denominators are not greater than 100; and it was remarked "There seems to be no doubt that this is the form of Brocot's original table." We now find that the tables are practically identical in form except that the Brocot table is to $10 \mathrm{D}$, instead of to $11 \mathrm{D}$. The table occupies p. 47-89, and pages $91-97$ are in English, the half title being "Use of the table for the calculation of clock-trains by approximation." Brocot tells us that this section was "specially intended for English clock-makers." Compare RMT 87.

$$
\text { R. C. A. }
$$

\section{CORRIGENDA ET ADDENDUM}

P. 6, 54, for circular and cosine, read circular sine and cosine.

P. 19, 1. 15 from bottom, for Martha Jaeger, read Martha Clarke. P. 33, 1. 11 and p. 100, for Theodericus, read Theodoricus. P. 40, 1. 3, for 639000, read 63900. P. 42, 1. 6, for 1848 , read 1858. P. 44, footnote 7, 1. 1, for $g$, , ", read g, ', ". P. 45, 1. 26, for 1,0000, read 1.0000 ; for 1.000 , read 1.0000 ; for 25000 , read 2.5000 .

P. 46, 1. 10, for 0:00100, read 0.00100. P. 50, for $e^{-i x}$, read $e^{-i x^{2}} ;$ for $d x$, read $d x^{n} ;$ for $h(x)$, read $H(x)$; for and $H(0.3)$, read and $H(0.2)$. P. $51,1.7$, for Levinson, read Levenson. P. 55, 1. 7, for 6.974, read 6.474. P. 61 MAC, 1. 11, for specializing, read specialize. P. $70,1909,1.2$, read 100(100)1000; 1912 for specimal, read specimen. P. 71, 1914, 1.3 read $C_{n}{ }^{\prime} \equiv d C_{n}(x) / d x$. P. 72, 34, for A. E. KenNelly, read P. L. AlgeR.

P. 74, 54, 1. 3, read $J_{3}(x) / I_{3}(x)$. P. 75, 1.5 from bottom, for .6(.2)1, read .6(.02)1. P. 76, 1. 11, for $(.005) 1(.001) \cdot 9$, read (.005).1(.001).9. P. 84, (7 c.s.), read . . huit Décimales des Nombres entiers de 1 d 120000 et des Sinus et Tangentes de dix Secondes en dix Secondes d'Arc . . .; (11 t.s.), 1. 3, for 1s, read 10s. P. 75, for Kennelly, read Alger. P. 91, Table III, 1. 5, for $-7.0^{*}$, read $-70^{*}$.

P. 94, 1. 5, from bottom for $0(.001) .99$, read $0(.0001) .99$. P. $95,1.5$, for $5 \mathrm{D}$, read to degrees and minutes; 1.13 , for $4 \mathrm{~S}$, read $4 \mathrm{D} ; 1$. 20, read Umkehrfunktionen. $\mathrm{P} .96$, for $\mathbf{5}(\mathrm{X})$, read $\mathbf{5}(\mathrm{Z})$. P. 99, 1. 13, for $0(0.01) 3$, read $0(0.1) 3$.

P. 69, 1879, add to the described contents of Glaisher's article as follows: On p. 53 are given the zeros of $P_{n}(x)$, for $n=[2(1) 7 ; 15 \mathrm{D}]$. 